\title{
Making Adaptive an Interval Constraint Propagation Algorithm Exploiting Monotonicity
}

\author{
Ignacio Araya, Gilles Trombettoni, Bertrand Neveu \\ UTFSM (Chile), INRIA, Univ. Nice-Sophia, Imagine LIGM Univ. Paris-Est (France) \\ iaraya@inf .utfsm.cl, \{Gilles.Trombettoni, Bertrand.Neveu\}@sophia.inria.fr
}

\begin{abstract}
A new interval constraint propagation algorithm, called MOnotonic Hull Consistency (Mohc), has recently been proposed. Mohc exploits monotonicity of functions to better filter variable domains. Embedded in an interval-based solver, Mohc shows very high performance for solving systems of numerical constraints (equations or inequalities) over the reals. However, the main drawback is that its revise procedure depends on two user-defined parameters.

This paper reports a rigourous empirical study resulting in a variant of Mohc that avoids a manual tuning of the parameters. In particular, we propose a policy to adjust in an auto-adaptive way, during the search, the parameter sensitive to the monotonicity of the revised function.
\end{abstract}

\section{Introduction}

Interval-based solvers can solve systems of numerical constraints, i.e., nonlinear equations or inequalities over the reals. Their reliability and increasing performance make them applicable to various domains such as robotics design [10], dynamic systems in robust control or robot localization [8], robust global optimization $[7,12]$ and bounded-error parameter estimation [6].

To find all the solutions of a numerical CSP, an interval-based solving strategy starts from an initial search space called a box (an interval for every variable in the handled system) and builds a search tree by following a Branch $\& 6$ Contract scheme. Filtering or contraction algorithms reduce the search space, i.e., improve the bounds of the corresponding variable intervals, with no loss of solution. Mohc is a new contraction algorithm based on interval constraint propagation [2]. Mohc-Revise exploits monotonicity of functions to improve contraction/filtering. Monotonicity is generally verified for a few functions at the top of the search tree, but can be detected for more functions when smaller boxes are handled. In practice, experiments shown in [2,1] highlight very high performance of Mohc, in particular when it is used inside 3BCID [13]. The combination 3BCID (Mohc) appears to be state of the art in this area.

The Mohc-Revise algorithm handles two user-defined parameters falling in $[0,1]$ : a precision ratio $\epsilon$ and another parameter called $\tau_{m o h c}$ that reflects a sensitivity to the degree of monotonicity of the handled function $f$. This paper mainly presents a rigourous empirical study leading to a policy for tuning $\tau_{m o h c}$ 
in an adaptive way for every function $f$. An adjustment procedure is run in a preprocessing phase called at every node of the search tree. Its cost is negligible when Mohc is called in combination with 3BCID.

\section{Intervals and numerical CSPs}

Intervals allow reliable computations on computers by managing floating-point bounds and outward rounding.

\section{Definition 1 (Basic definitions, notations)}

$\mathbb{I} \mathbb{R}$ denotes the set of intervals $[v]=[a, b] \subset \mathbb{R}$, where $a$, also denoted $\underline{v}$, and $b$, also denoted $\bar{v}$, are floating-point numbers.

$\operatorname{Diam}([v]):=\bar{v}-\underline{v}$ denotes the size, or diameter, of $[v]$.

$A$ box $[V]=\left(\left[v_{1}\right], \ldots,\left[v_{n}\right]\right)$ represents the Cartesian product $\left[v_{1}\right] \times \ldots \times\left[v_{n}\right]$.

Interval arithmetic has been defined to extend to $\mathbb{R}$ elementary functions over $\mathbb{R}[11]$. For instance, the interval sum is defined by $\left[v_{1}\right]+\left[v_{2}\right]=\left[\underline{v_{1}}+\right.$ $\left.\underline{v_{2}}, \overline{v_{1}}+\overline{v_{2}}\right]$. When a function $f$ is a composition of elementary functions, an extension of $f$ to intervals $\mathbb{I} \mathbb{R}$ must be defined to ensure a conservative image computation. The natural extension $[f]_{N}$ of a real function $f$ replaces the real arithmetic by the interval arithmetic. The monotonicity-based extension is particularly useful in this paper. A function $f$ is monotonic w.r.t. a variable $v$ in a given box $[V]$ if the evaluation of the partial derivative of $f$ w.r.t. $v$ is positive (resp. negative) or null in every point of $[V]$. For the sake of conciseness, we sometimes write that the variable $v$ is monotonic.

Definition 2 ( $f_{\min }, f_{\max }$, monotonicity-based extension)

Let $f$ be a function defined on variables $V$ of domains $[V]$. Let $X \subseteq V$ be a subset of monotonic variables. Consider the values $x_{i}^{+}$and $x_{i}^{-}$such that: if $x_{i} \in X$ is an increasing (resp. decreasing) variable, then $x_{i}^{-}=x_{i}$ and $x_{i}^{+}=\overline{x_{i}}$ (resp. $x_{i}^{-}=\overline{x_{i}}$ and $x_{i}^{+}=x_{i}$ ). Consider $W=V \backslash X$ the set of variables not detected monotonic. Then, we define

$$
\begin{aligned}
& f_{\min }(W)=f\left(x_{1}^{-}, \ldots, x_{n}^{-}, W\right) \\
& f_{\max }(W)=f\left(x_{1}^{+}, \ldots, x_{n}^{+}, W\right)
\end{aligned}
$$

Finally, the monotonicity-based extension $[f]_{M}$ of $f$ in the box $[V]$ produces the interval image $[f]_{M}([V])=\left[\underline{\left[f_{\min }\right]_{N}([W])}, \overline{\left[f_{\max }\right]_{N}([W])}\right]$.

Consider for example $f\left(x_{1}, x_{2}, w\right)=-x_{1}^{2}+x_{1} x_{2}+x_{2} w-3 w$. $[f]_{N}([6,8],[2,4],[7,15])=-[6,8]^{2}+[6,8] \times[2,4]+[2,4] \times[7,15]-3 \times[7,15]=$ $[-83,35] \cdot \frac{\partial f}{\partial x_{1}}\left(x_{1}, x_{2}\right)=-2 x_{1}+x_{2}$. Since $\left[\frac{\partial f}{\partial x_{1}}\right]_{N}([6,8],[2,4])=[-14,-8]<0$, we deduce that $f$ is decreasing w.r.t. $x_{1}$. With the same reasoning, $x_{2}$ is increasing and $w$ is not detected monotonic. Following Def. 2, the monotonicity-based evaluation yields:

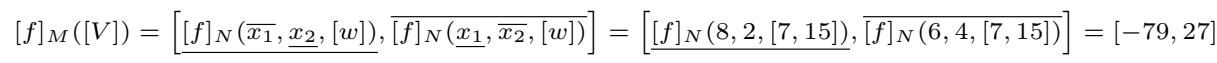


The dependency problem is the main issue of interval arithmetic. It is due to multiple occurrences of a same variable in an expression that are handled as different variables by interval arithmetic and produce an overestimation of the interval image. Since the monotonicity-based extension replaces intervals by bounds, it suppresses the dependency problem for the monotonic variables. It explains why the interval image computed by $[f]_{M}$ is sharper than or equal to the one produced by $[f]_{N}$.

This paper deals with nonlinear systems of constraints or numerical CSPs.

Definition 3 (NCSP) $A$ numerical CSP $P=(V, C,[V])$ contains a set of constraints $C$, a set $V$ of $n$ variables with domains $[V] \in \mathbb{I R}^{n}$.

$A$ solution $S \in[V]$ to $P$ satisfies all the constraints in $C$.

The interval-based solving strategies follow a Branch $\&$ Contract process to find all the solutions of an NCSP. We present two existing interval constraint propagation algorithms for contracting the current box in the search tree with no loss of solution. HC4 and Box [3,14] perform a constraint propagation loop and differ in their revise procedure handling the constraints individually. The procedure HC4-Revise traverses twice the tree representing the mathematical expression of the constraint for narrowing all the involved variable intervals. Since the different occurrences of a same variable are handled as different variables, HC4-Revise is far from bringing an optimal contraction (dependency problem). The BoxNarrow revise procedure of Box is more costly and stronger than HC4-Revise [5]. For every pair $(f, x)$, where $f$ is a function of the considered NCSP and $x$ is a variable involved in $f$, BoxNarrow first replaces the other $a$ variables in $f$ by their interval $\left[y_{1}\right], \ldots,\left[y_{a}\right]$. Then, it uses a shaving principle where slices $\left[x_{i}\right]$ at the bounds of $[x]$ that do not satisfy the constraint are eliminated from $[x]$. BoxNarrow is not optimal in case the variables $y_{i}$ different from $x$ also have multiple occurrences.

These algorithms are used in our experiments as sub-contractor of 3BCID [13], a variant of $3 \mathrm{~B}[9]$. 3B uses a shaving refutation principle that splits an interval into slices. A slice at the bounds is discarded if calling a sub-contractor (e.g., HC4) on the resulting subproblem leads to no solution.

\section{Overview of the Mohc algorithm}

The MOnotonic Hull-Consistency algorithm (in short Mohc) is a recent constraint propagation algorithm that exploits monotonicity of functions to better contract a box [2]. The contraction brought by Mohc-Revise is optimal (with a precision $\epsilon)$ when $f$ is monotonic w.r.t. every variable $x$ involved in $f$ in the current box. Mohc has been implemented with the interval-based C++ library Ibex [4]. It follows a standard propagation loop and calls the Mohc-Revise procedure for handling one constraint $f(V)=0$ individually (see Algorithm 1).

Mohc-Revise starts by calling HC4-Revise. The monotonicity-based contraction procedures (i.e., MinMaxRevise and MonotonicBoxNarrow) are then called only if $V$ contains at least one variable that appears several times (function 


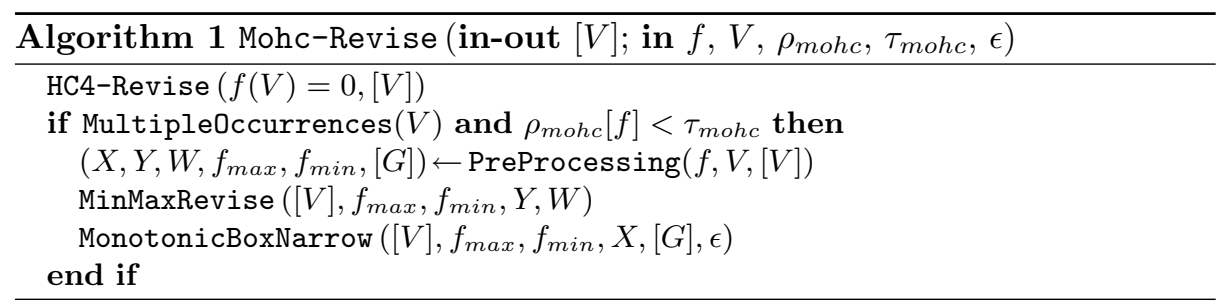

Multiple0ccurrences). The other condition is detailed below. The procedure PreProcessing computes the gradient of $f$ (stored in the vector $[G]$ ) and determines the two functions $f_{\min }$ and $f_{\max }$, introduced in Definition 2, that exploit the monotonicity of $f$. The gradient is also used to partition the variables in $V$ into three subsets $X, Y$ and $W$ :

- variables in $X$ are monotonic and occur several times in $f$,

- variables in $Y$ occur once in $f$ (they may be monotonic),

- variables $w \in W$ appear several times in $f$ and are not detected monotonic, i.e., $0 \in\left[\frac{\partial f}{\partial w}\right]_{N}([V])$. (They might be monotonic - due to the overestimation of the evaluation - but are considered and handled as non monotonic.)

The next two routines are in the heart of Mohc-Revise. Using the monotonicity of $f_{\min }$ and $f_{\max }$, MinMaxRevise contracts $[Y]$ and $[W]$ while MonotonicBoxNarrow contracts $[X]$. MinMaxRevise is a monotonic version of HC4-Revise. It applies HC4-Revise on the two constraints: $f_{\min }([Y \cup W]) \leq 0$ and $0 \leq f_{\max }([Y \cup W])$. MonotonicBoxNarrow performs a loop on every monotonic variable $x \in X$. If $f$ is increasing w.r.t. $x$, it performs a first binary search with $f_{\max }$ to improve $\underline{x}$, and a second one with $f_{\min }$ to improve $\bar{x}$. A binary search runs in time $O\left(\log \left(\frac{1}{\epsilon}\right)\right)$, where $\epsilon$ is a user-defined precision parameter expressed as a percentage of the interval size.

The user-defined parameter $\tau_{m o h c} \in[0,1]$ allows the monotonicity-based procedures to be called more or less often during the search (see Algorithm 1). For every constraint, the procedures exploiting monotonicity of $f$ are called only if $\rho_{\text {mohc }}[f]<\tau_{\text {mohc }}$. The ratio $\rho_{\text {mohc }}[f]=\frac{\operatorname{Diam}\left([f]_{M}([V])\right)}{\operatorname{Diam}\left([f]_{N}([V])\right)}$ indicates whether the monotonicity-based image of a function is sufficiently sharper than the natural one. $\rho_{m o h c}[f]$ is computed in a preprocessing procedure called after every bisection/branching. Since more cases of monotonicity occur on smaller boxes, Mohc-Revise activates in an adaptive way the machinery related to monotonicity. This ratio is relevant for the bottom-up evaluation phases of MinMaxRevise, and also for MonotonicBoxNarrow in which a lot of evaluations are performed.

\section{Making Mohc auto-adaptive}

The procedure Mohc-Revise has two user-defined parameters, whereas BoxNarrow has one parameter and HC4-Revise has no parameter. The goal of this paper is to avoid the user fixing the parameters of Mohc-Revise. 
All the experiments have been conducted on the same Intel $66002.4 \mathrm{GHz}$ over 17 NCSPs with a finite number of zero-dimensional solutions issued from COPRIN's web page maintained by J.-P. Merlet. ${ }^{1}$ Ref. [2] details the criteria used to select these NCSPs with multiple occurrences. ${ }^{2}$ All the solving strategies use a round-robin variable selection. Between two branching points, three procedures are called in sequence. First, a monotonicity-based existence test [1] cuts the current branch if the image computed by a function does not contain zero. Second, the evaluated contractor is called : 3BCID (Mohc) or 3BCID (Amohc) where Amohc denotes an auto-adaptive variant of Mohc. Third, an interval Newton is run. The shaving precision ratio in 3BCID is 10\% ; a constraint is pushed into the propagation queue if the interval of one of its variables is reduced more than $10 \%$.

We have first studied how the ratio $\frac{\operatorname{Time}(3 B C I D(\text { Mohc }))}{\operatorname{Time}(3 B C I D(\operatorname{LazyMohc}))}$ evolves when $\epsilon$ decreases, i.e., when the required precision in MonotonicBoxNarrow increases. LazyMohc [1] is a variant of Mohc that does not call MonotonicBoxNarrow. The measured ratio thus underlines the additional contraction brought by this binary search. For all the tested instances, the best value of $\epsilon$ falls between $\frac{1}{32}$ and $\frac{1}{8}$, which led us to fix $\epsilon$ to $10 \%$. Further decreasing $\epsilon$ turns out to be a bad idea since the ratio remains quasi-constant (see Fig. 4.4-left in [1]).

The main contribution of this paper is an empirical analysis that has led us to an automatic tuning of the $\tau_{m o h c}$ parameter during the search.

Table 1 contains the main results useful for our analysis. It reports the CPU time (first row of a multi-row) required by the solving strategy described above (based on 3BCID (Mohc)) in function of $\tau_{m o h c}$. The NCSPs are sorted by decreasing gains in performance of 3BCID (Amohc) w.r.t. 3BCID (HC4) (column G/3B). The entries of columns 2 to 10 also contain (second row of a multi-row) a gain falling in $[0,1]$. The gain is $\frac{\operatorname{Time}(3 B C I D(\text { Mohc }))}{\operatorname{Time}(3 B C I D(\text { Oracle }))}$, where Oracle is a theoretical Mohc variant that would be able to select the best value (yielding the results in bold in the table) for $\tau_{m o h c}$.

The first 10 columns highlight that the best value of $\tau_{m o h c}$ nearly always falls in the range $[50 \%, 99.99 \%]$. The value $50 \%$ yields always better results than (or similar to) $\tau_{m o h c}=40 \%$ or less [1]. Also, $\tau_{m o h c}=99.99 \%$ is better than or similar to $100 \%$ (except in ButcherA). Note that Mohc with $\tau_{m o h c}=0 \%$ is identical to HC4 (see Algorithm 1).

Second, a significant correlation can be observed on Table 1 and on the graphic shown in Fig. 1. The curves show how the application frequency of the monotonicity-based procedures $\left(\frac{\text { number_of_calls }\left(\rho_{m o h c}[f]<\tau_{m o h c}\right)}{\text { number_of_calls }(\text { Mohc }-\operatorname{Revise})}\right)$ evolves when $\tau_{m o h c}$ increases. (Of course, the frequency becomes 1 when $\tau_{m o h c}=1$.) The correlation can be observed vertically for any value of $\tau_{m o h c}$, although this is clearer for intermediate values. For instance, with the abscissa $\tau_{m o h c}=60 \%$,

\footnotetext{
${ }^{1}$ See www-sop.inria.fr/coprin/logiciels/ALIAS/Benches/benches.html

2 Compared to [2], the NCSP kin1 has been removed because it can be solved with 3BCID (HC4) in less than 100 choice points. ButcherB has been added. ButcherA (with $[a]=[-50,-1.1]$ ) and ButcherB (with $[a]=[-0.9,50]$ ) are two sub-instances of the NCSP Butcher. Merlet has manually removed the value $a=1$ to avoid a singularity...
} 
Table 1. Experimental results. The column $G / 3 B=\frac{\operatorname{Time}(3 B C I D(H C 4))}{\operatorname{Time}(3 B C I D(A m o h))}$ highlights the gain of our 3BCID (Amohc) contractor w.r.t. the standard 3BCID (HC4) (column 2).

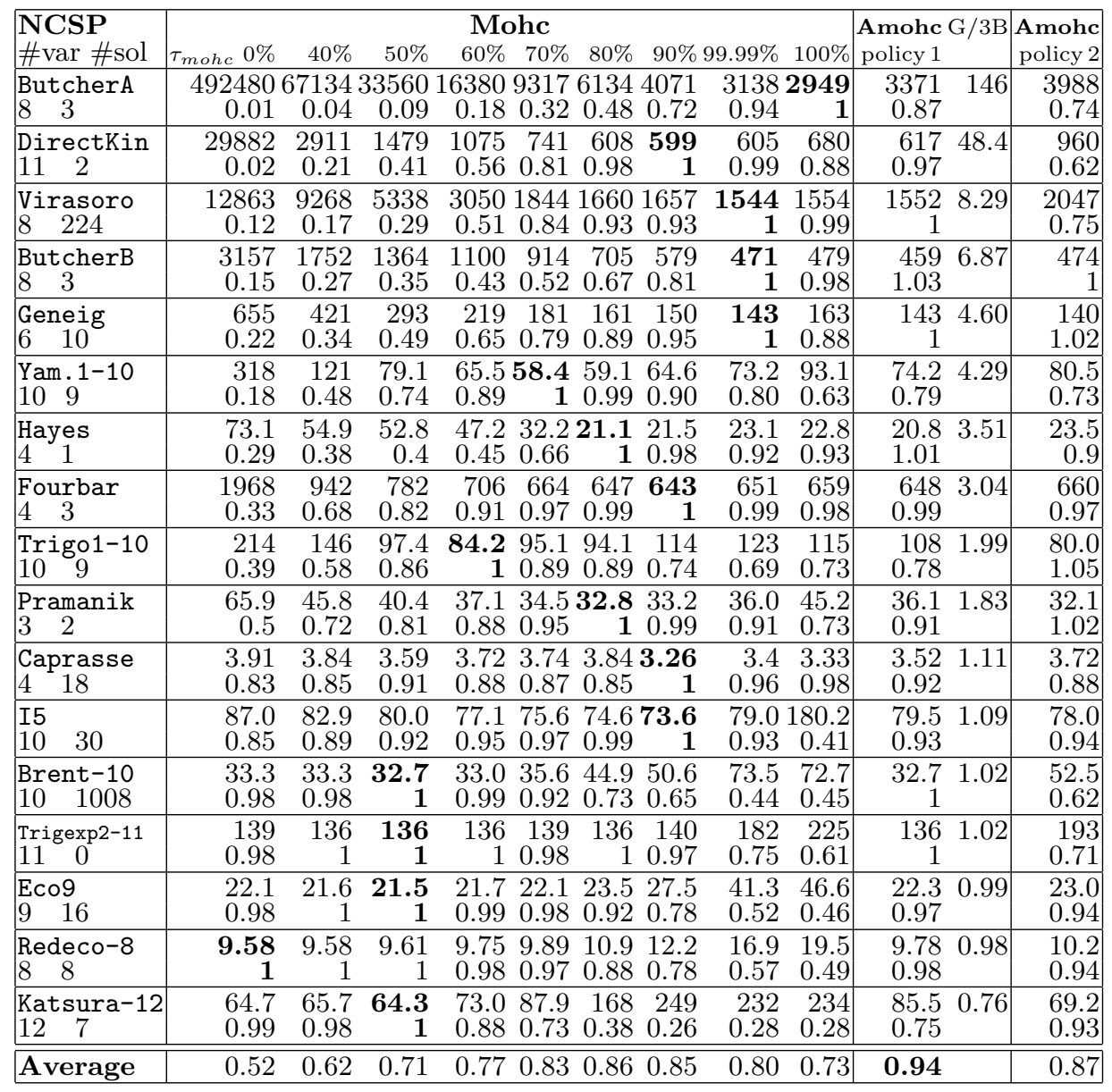

the application frequencies of Katsura, Redeco, Eco9, Trigexp2, Brent and I5 are inferior to $10 \%$. It appears that these instances are not very well solved by 3BCID (Mohc) with $\tau_{m o h c}=100 \%$ (compared to 3BCID(HC4)). This suggests that when the application frequency is low (resp. high), $\tau_{m o h c}$ should rather be tuned to a low (resp. high) value. Intuitively, a high frequency means that a lot of monotonicity-based evaluations produce a sharp interval image, so that Mohc should well exploit these sharp evaluations.

This study has led us to a simple auto-adaptive $\tau_{m o h c}$ tuning policy following three significant choices:

- Since Mohc-Revise exploits the monotonicity of a single function $f$, there is no reason that $\tau_{m o h c}$ be the same for all constraints. This prevented the user 

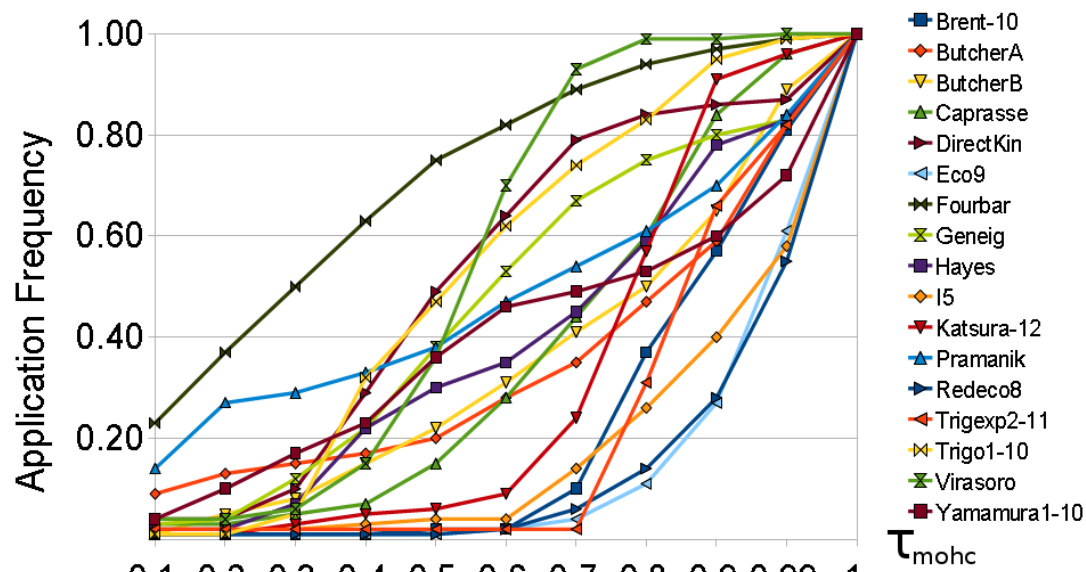

$\begin{array}{lllllllllll}0.1 & 0.2 & 0.3 & 0.4 & 0.5 & 0.6 & 0.7 & 0.8 & 0.9 & 0.99 & 1\end{array}$

Fig. 1. Application frequency of the monot. based procedures in function of $\tau_{m o h c}$.

from specifying a $\tau_{m o h c}$ parameter for each function $f$, but this simplification is no more relevant in an adaptive tuning policy. ${ }^{3}$

- For any constraint $f, \tau_{m o h c}[f]$ is fixed to one of both values $50 \%$ and $99.99 \%$. Indeed, if we had an oracle able to select in any instance the best value for $\tau_{m o h c}$ among $50 \%$ and $99.99 \%$, the loss in performance w.r.t. an oracle knowing the best value of $\tau_{m o h c}$ would be only $4 \% . \tau_{m o h c}=50 \%$ (resp. $99.99 \%$ ) is generally the most relevant value when the gain in CPU time of 3BCID (Mohc) compared to 3BCID (HC4) is the smallest (resp. greatest).

- The application frequency is updated at each node of the search tree. It is more and more accurate as the number of measurements increases.

These choices lead to the tuning policy 1 based on Algorithm 2. The procedure ComputeRhoMohc is run for every constraint at each node of the search tree, before running the contraction procedures. nb_calls [f] and nb_interesting [f], related to a given constraint $f$, are initialized to 0 before the search. The ratios tau_mohc [f] are set to $99.99 \%$ during the first 50 nodes before being adjusted in ComputeRhoMohc. The results are not sensitive to a fine tuning of RHO_INTERESTING and TAU_FREQ, empirically fixed to $65 \%$ and $10 \%$ respectively.

The performance of this policy is illustrated by the last line of Table 1 . The loss in performance w.r.t. an oracle knowing the best value of $\tau_{m o h c}\left(\tau_{m o h c}\right.$ being common to all the constraints in a given NCSP) is $6 \%$ on average (at worst $25 \%$; around $0 \%$ in 7 NCSPs). The obtained average gain (94\%) highlights that Amohc is better than any fixed value of $\tau_{m o h c}$ (i.e., $86 \%$ for $\tau_{m o h c}=80 \%$ ). The column $\mathrm{G} / 3 \mathrm{~B}$ is also very convincing because 3BCID (Amohc) and 3BCID (HC4) have the same number of parameters, i.e., 0 coming from HC4-Revise and Amohc-Revise.

3 This explains why our auto-adaptive versions of Mohc sometimes (slightly) outperform the oracle so that the corresponding gains in the table are greater than 1. 


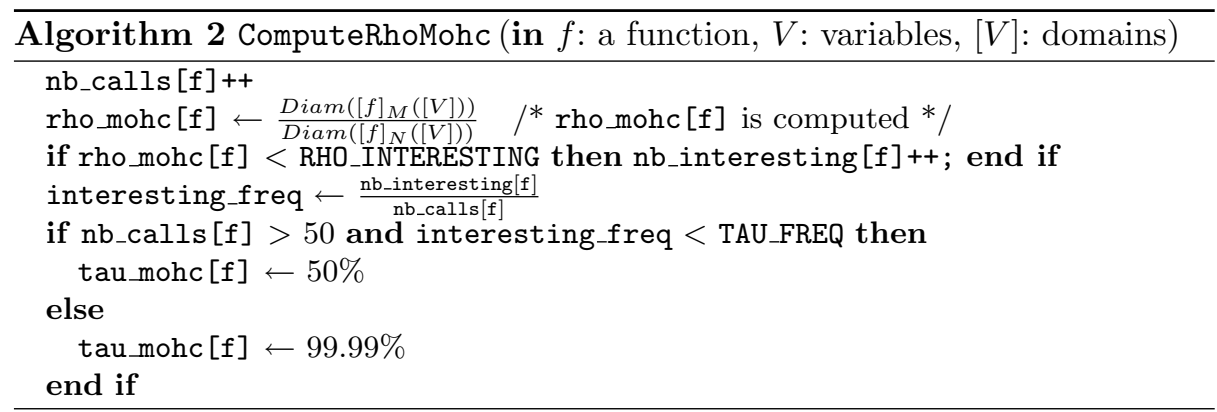

An alternative policy 2 is based on contraction. Each time the monotonicitybased procedures of Algorithm 1 are applied, two ratios of box perimeters are computed: $r_{e}$ is the gain in perimeter brought by HC4-Revise w.r.t. the initial box; $r_{m}$ is the gain brought by MinMaxRevise+MonotonicBoxNarrow w.r.t. the previous box. If $r_{m}$ is better (enough) than $r_{e}$, then $\tau_{m o h c}$ is slightly incremented; if $r_{e}$ is better than $r_{m}$, then it is decremented.

As shown in Table 1, the policy 2 is not so bad although less efficient than the policy 1 on average (87\%). Unfortunately, this adaptive policy is the worst when Mohc is the most useful (e.g., see ButcherA, DirectKin, Virasoro).

\section{References}

1. I. Araya. Exploiting Common Subexpressions and Monotonicity of Functions for Filtering Algorithms over Intervals. PhD thesis, University of Nice-Sophia, 2010.

2. I. Araya, G. Trombettoni, and B. Neveu. Exploiting Monotonicity in Interval Constraint Propagation. In Proc. AAAI (to appear), 2010.

3. F. Benhamou, F. Goualard, L. Granvilliers, and J.-F. Puget. Revising Hull and Box Consistency. In Proc. ICLP, pages 230-244, 1999.

4. G. Chabert. www.ibex-lib.org, 2010.

5. H. Collavizza, F. Delobel, and M. Rueher. Comparing Partial Consistencies. Reliable Comp., 5(3):213-228, 1999.

6. L. Jaulin. Interval Constraint Propagation with Application to Bounded-error Estimation. Automatica, 36:1547-1552, 2000.

7. R. B. Kearfott. Rigorous Global Search: Continuous Problems. Kluwer, 1996.

8. M. Kieffer, L. Jaulin, E. Walter, and D. Meizel. Robust Autonomous Robot Localization Using Interval Analysis. Reliable Computing, 3(6):337-361, 2000.

9. O. Lhomme. Consistency Tech. for Numeric CSPs. In IJCAI, pages 232-238, 1993.

10. J-P. Merlet. Interval Analysis and Robotics. In Symp. of Robotics Research, 2007.

11. R. E. Moore. Interval Analysis. Prentice-Hall, 1966.

12. M. Rueher, A. Goldsztejn, Y. Lebbah, and C. Michel. Capabilities of Constraint Programming in Rigorous Global Optimization. In NOLTA, 2008.

13. G. Trombettoni and G. Chabert. Constructive Interval Disjunction. In Proc. CP, LNCS 4741, pages 635-650, 2007.

14. P. Van Hentenryck, L. Michel, and Y. Deville. Numerica : A Modeling Language for Global Optimization. MIT Press, 1997. 